\title{
Well differentiated (benign) papillary mesothelioma of the tunica vaginalis
}

\author{
R Chetty
}

\begin{abstract}
An unusual mesothelial lesion occurred in the tunica vaginalis of the testis. It conformed histologically and immunohistochemically to well differentiated papillary mesothelioma of the peritoneum. Its aetiology remains uncertain, but this lesion, more than likely, is innocuous. It is important to recognise this entity, which is not well documented in the tunica vaginalis, because it may be misdignosed as a malignant mesothelioma and the patient may be subjected to unnecessary treatment.
\end{abstract}

(F Clin Pathol 1992;45:1029-1030)

Mesothelial lesions of the tunica vaginalis of the testis include mesothelial hyperplasia, adenomatoid tumour, and frankly malignant mesothelioma. Well differentiated papillary mesothelioma (WDPM) also falls in the ambit of mesothelial proliferations of the tunica vaginalis, probably at the benign end of the spetrum. ${ }^{1}$ This uncommon mesothelial lesion is seen more commonly in the peritoneum of young women ${ }^{1}$ and very rarely in the tunica vaginalis. $^{23}$

\section{Case report}

An 18 year old man complained of a scrotal swelling that had been gradually increasing in size over a period of weeks. There was no history of trauma or exposure to asbestos. A large right hydrocele was found on examination. No testicular masses were palpated. Systemic enquiry and examination yielded normal results. Initial aspiration produced about $150 \mathrm{ml}$ of straw-coloured fluid. However, the hydrocele reaccumulated, and surgery was performed. Two nodules, both measuring $1.0 \mathrm{~cm}$ in maximal diameter, were excised from the tunica vaginalis. Twelve months later the patient was well and asymptomatic.

\section{Pathological findings}

Both lesions were identical. A tubulo-papillary pattern dominated. The papillary structures had broad fibrous cores clothed by a single layer of cuboidal mesothelial cells (fig 1). Cytologically, these cells were uniformly bland and no mitotic activity was noted. Other areas of the lesion comprised a solid, cellular component. Here the cells were vacuolated and resembled those of an adenomatoid tumour. The stroma tended to be sclerotic with entrapment of mesothelial cells, producing a pseudoinfiltrative pattern. Typical psammoma bodies were also present (fig 2). No evidence of inflammation or haemosiderin deposits was seen.

The tumour cells contained vacuoles of

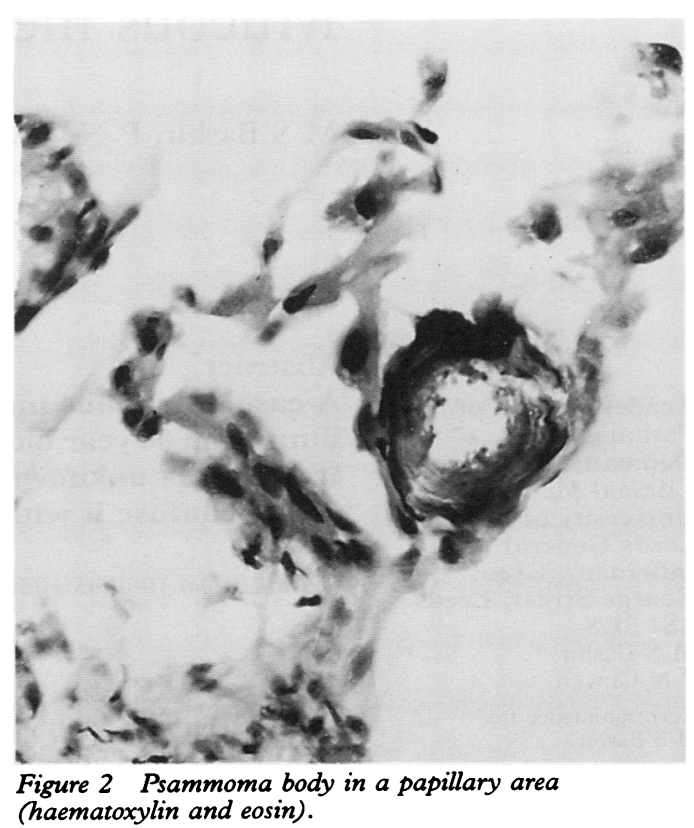


hyaluronic acid (alcian blue positivity digested by hyaluronidase).

Immunohistochemical examination of the tumour showed cytokeratin (CAM 5.2) and epithelial membrane antigen (EMA) positivity. Carcinoembryonic antigen (CEA) and Leu M-1 antibody were not expressed.

\section{Discussion}

The tunica vaginalis is lined by mesothelium: a full spectrum of mesothelial proliferations from completely benign to overtly malignant can occur.

Reactive hyperplasia of the tunica vaginalis may be attended by papillary change ${ }^{4}$ but this is usually a focal finding and not the dominant histological configuration.

More importantly, this lesion needs to be distinguished from malignant mesothelioma and papillary carcinoma, which display nuclear pleomorphism, mitotic activity, and areas of stromal invasion. In papillary areas multilayering of the epithelium is allied to the cytological atypia. Well differentiated malignant mesothelioma may have foci of well developed tubulopapillary structures devoid of cellular atypia. However, such areas are invariably accompanied by others showing obvious malignant features. The key microscopic feature of WDPM is the lack of cellular and architectural atypia.

In their analysis of 22 such tumours occurring in the peritoneum Daya and McCaughey found mitoses in only one case (the number not being quantified). ${ }^{1}$ Six cases also occurring in the peritoneum described by Goepel ${ }^{5}$ did not show mitotic activity. The case reported by Johnson, Fuerst, and Gallager in the tunica vaginalis of a 23 year old man, would seem to have had all the morphological characteristics of a WDPM. ${ }^{3}$ In this case mitoses were described as "infrequent", and no recurrence was noted after follow up for a year. ${ }^{3}$ No mitoses were present in the case of Barbera and Rubin, ${ }^{2}$ and the patient was well after one year.

Well differentiated papillary mesothelioma is usually a small lesion in both the peritoneum and tunica vaginalis. ${ }^{12}$ Most occur in the peritoneum, and very rarely in the tunica vaginalis, with only two definite cases having been reported so far. ${ }^{23}$ Other sites include the epicardium ${ }^{6}$ and pleura. ${ }^{7}$ Peritoneal lesions are more common in females, but cases have been described in the peritoneum in males.

Clinically, this lesion seems to run a bland course, but a course more akin to low grade malignancy in the peritoneum has not been entirely ruled out. ${ }^{1}$ With this uncertainty regarding their behaviour, these lesions are designated "well differentiated" rather than "benign".

Probably not associated with exposure to asbestos, the precise aetiology is not clear. Surgical excision with regular follow up is the current management.

1 Daya D, McCaughey WTE. Well differentiated papillary mesothelioma of the peritoneum. A clinicopathologic study of 22 cases. Cancer 1990;65:292-6.

2 Barbera V, Rubino M. Papillary mesothelioma of the tunica vaginalis. Cancer 1957;10:183-9.

3 Johnson DE, Fuerst DE, Gallager HS. Mesothelioma of the tunica vaginalis. South Med $\mathcal{F}$ 1973;66:1295-7.

4 Foyle A, Al-Jabi M, McCaughey WTE. Papillary peritonea tumors in women. Am $\mathcal{f}$ Surg Pathol 1981;5:241-9.

5 Goepel JR. Benign papillary mesothelioma of peritoneum: A histological, histochemical and ultrastructural study of six cases. Histopathology 1981;5:21-30.

6 Larsen TE. Serosal papiloma of the epicardium. Arch Pathol 1974;97:271-2.

7 Yesner R, Hurwitz A. Localised pleural mesothelioma of epithelial type. F Thorac Surg 1953;26:325-9.

8 Daya D, McCaughey WTE. Pathology of the peritoneum: A review of selected topics. Semin Diagn Pathol 1991;8:227-89.

\footnotetext{
Academic Unit of Pathology, Department of Clinical Medicine, University of Leeds, Leeds General Infirmary, Great George Street, Leeds LS1 3EX

M S Bashir

P N Cowen

Correspondence to:

M S Bashir

Accepted for publication 18 May 1992
}

\begin{abstract}
A case of mucous metaplasia of mesothelium in an 80 year old woman is described. Its cause is unknown, but it is important not to confuse it with secondary tumour.
\end{abstract}

(F Clin Pathol 1992;45:1030-1031)

\section{Case report}

An 80 year old woman presented with upper abdominal pain and weight loss. A right sided pleural effusion was found on examination but

\title{
Mucous metaplasia of the pleura
}

\author{
M S Bashir, P N Cowen
}

no abdominal signs were detected clinically or on ultrasound scan. She had never complained of respiratory symptoms, and a chest $x$-ray picture after pleurocentesis, when pleural biopsy was performed, was normal. There was nothing to suggest new growth in this patient and she died about a week later. Necropsy was not performed and cause of death was given as cardiorespiratory failure.

\section{Pleural histology}

This showed inflamed vascular connective 